\title{
Trafficability of rigid wheel with bionic lug on sand by 3D DEM
}

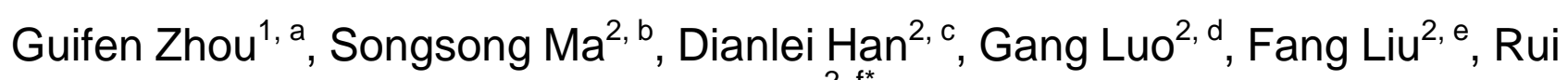 \\ Zhang ${ }^{2, f^{*}}$
}

${ }^{1}$ College of Engineering and Technology, Jilin Agricultural University, Changchun, 130118, China

${ }^{2}$ Key Laboratory of Bionic Engineering, Ministry of Education, Jilin University, Changchun, 130022, China

aemail: guifenzhou@163.com, ${ }^{\text {bemail: 604188594@qq.com, }{ }^{c} e m a i l: ~ 1553916045 @ q q . c o m, ~}$

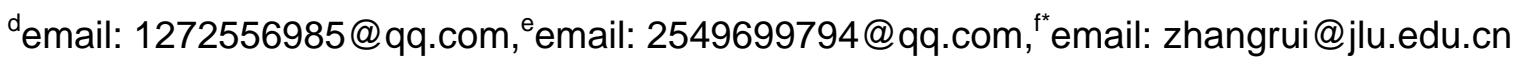

Keywords: rigid wheel with bionic lug, trafficability on sand, PFC3D, DEM simulation

\begin{abstract}
Numerical simulation has significant advantage in analyzing the interaction between rigid wheel and lunar soil simulants and designing the lunar vehicle wheel under complex circumstances. The computer aided design software CATIA and 3D Discrete Element Method simulation software PFC3D were combined by the interface codes and the embedded FISH language in PFC3D. Then, this method was used to establish the dynamic simulation system of interactions between the wheel with lugs and loose lunar soil stimulants. The dynamic simulation system was validated by the wheel soil-bin test and then was utilized to simulate that the wheel with bionic lug and the wheel with rectangle lug travel on lunar soil simulants with different slip rate. The result indicated that wheel with bionic lug had larger traction and well stability than wheel with rectangle lug.
\end{abstract}

\section{Introduction}

The rover is the important equipment for landing on the moon and accomplishing lunar exploration. The lunar surface is covered with loose powder regolith that greatly affects the lunar rover wheel passing ability. Therefore, it is particularly important to develop the lunar rover wheel with superior traction through lunar soil. At present, great attention of science researchers have been paid on the research of tractive characteristics of a wheel on the lunar regolith and wheel configuration optimization[1]. The rigid wheel with wheel lug has the high traction in loose sandy environment, and it has been an important choice of wheel configuration of desert travelling vehicle. Many creatures have formed excellent features of environmental adaption after natural evolutionary optimization. Nowadays these excellent characters of creatures were widely applied in bionic engineering [2-4]. Research found that animals living in the desert had superior ability of travelling across sand. Inspired by the claw of ostrich, a bionic lug was designed.

Discrete element method (DEM) is the discrete numerical method for analyzing discrete or granular materials. In DEM, the soil is regarded as the aggregates composed of many independent units. DEM has become an ideal tool for studying the interaction between rigid wheel and loose lunar soil simulants. At present, researchers have studied the wheel-soil interactions by DEM, and have obtained a series of important research results [5-6]. Yet, it is difficult to obtain the passing ability analyses of the rigid wheel with irregular structure in the loose lunar soil stimulant environment for most DEM software lack the ability of establishing the complex solid structure model. In order to save this problem, the computer aided design software CATIA and 3D DEM software PFC3D were combined in this paper.

\section{Design of bionic lug and rectangle lug on wheel}

The ostrich, specialized in running with a surprising speed, live in the plain and the desert. During running on sand, the toe nail contributes a lot for the high speed of ostrich because toe nail can enhance sand fixation effects and flow limitation. 
The structure of ostrich toe nail is very special (Fig. 1a, b).Reverse engineering was used to get the three-dimensional point cloud data which was then imported into CATIA (Fig. 1c). After removing noise points, filling up of loophole and simplifying point cloud, exported file was imported into NX 8.0 to get the contour line(Fig. 1d) for bionic design.

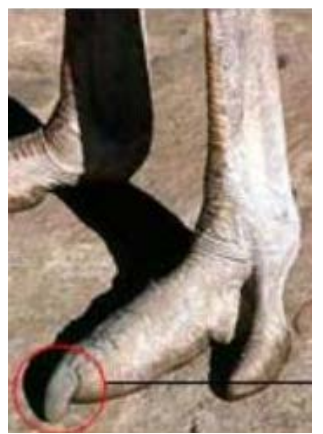

a

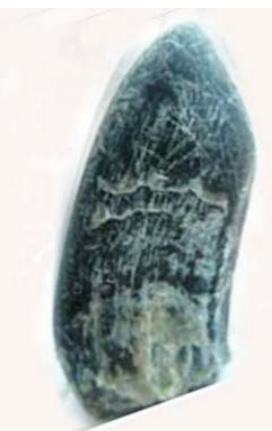

$b$

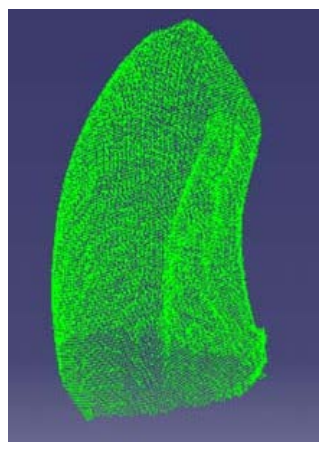

C

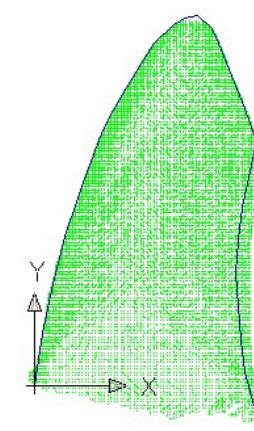

d

Fig. 1 Process of getting contour line for bionic design

The CAD modeling software CATIA was utilized to model the 3D structure of wheel with rectangle lugs (Fig. 2) and the wheel with bionic lugs(Fig. 3) that was designed based on ostrich claw.

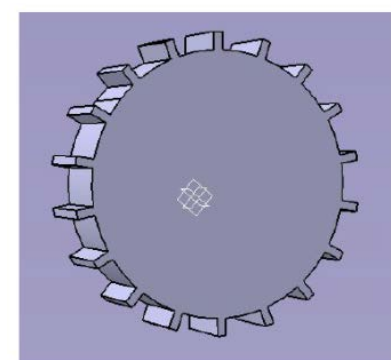

Fig. 2 The wheel with rectangle lugs

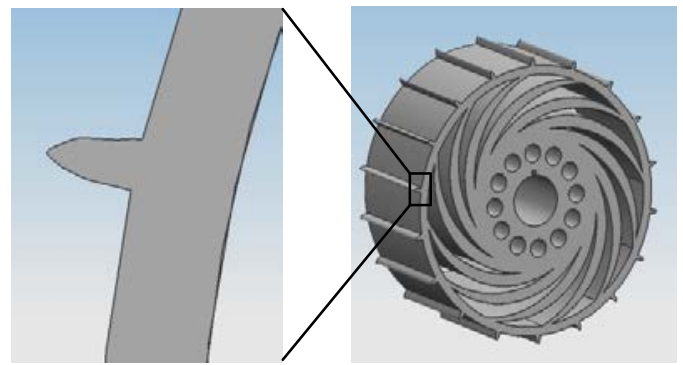

Fig. 3 The wheel with bionic lugs

\section{Establishing of simulation system}

Dynamic simulation system of the interaction between wheel and discrete lunar simulants was shown in Fig. 4. In the discrete element mechanical model of lunar soil simulants, the spring and the damper respectively represented elasticity and inelasticity of contact between particles of lunar soil simulants. In addition, the sliders with coefficient of friction represented friction among particles of lunar soil simulants. As shown in Fig. 4, this paper hypothesized that, between two disc shaped lunar soil simulants particles $\mathrm{i}$ and $\mathrm{j}$, there were a spring with normal elastic constant $k_{n}$ and tangential elastic constants $k_{s}$, a damper with normal damping $\eta_{n}$ and tangential damping $\eta_{s}$, a slider with coefficient friction $\mu$, and a non-tension connection representing inexistence of tension between one lunar soil simulants particle and other particles.

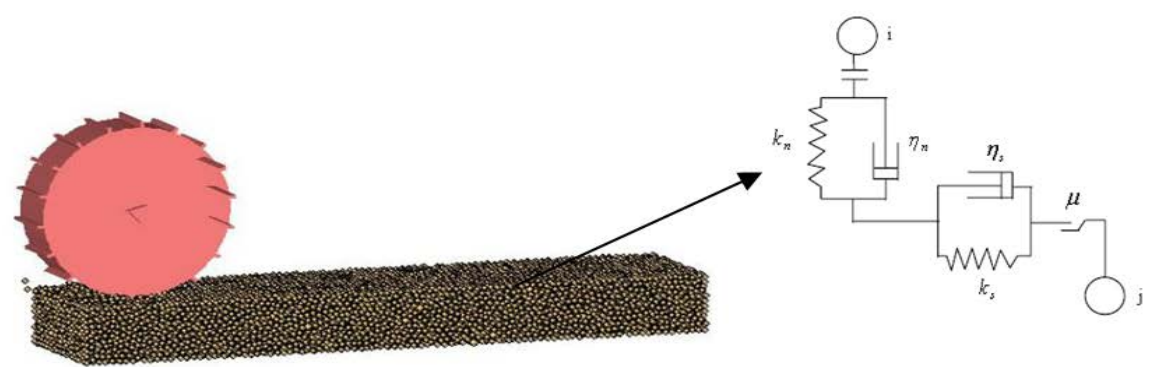

Fig. 4 Dynamic simulation system of interactions between irregular rigid wheel and lunar soil simulants 
As shown in Table 1, the model parameters of micro-contact was obtained after the repeated simulation and comparison, which can reflect mechanical property of lunar soil simulants.

Table 1. The mesoscopic mechanical parameters of lunar soil simulants in DEM

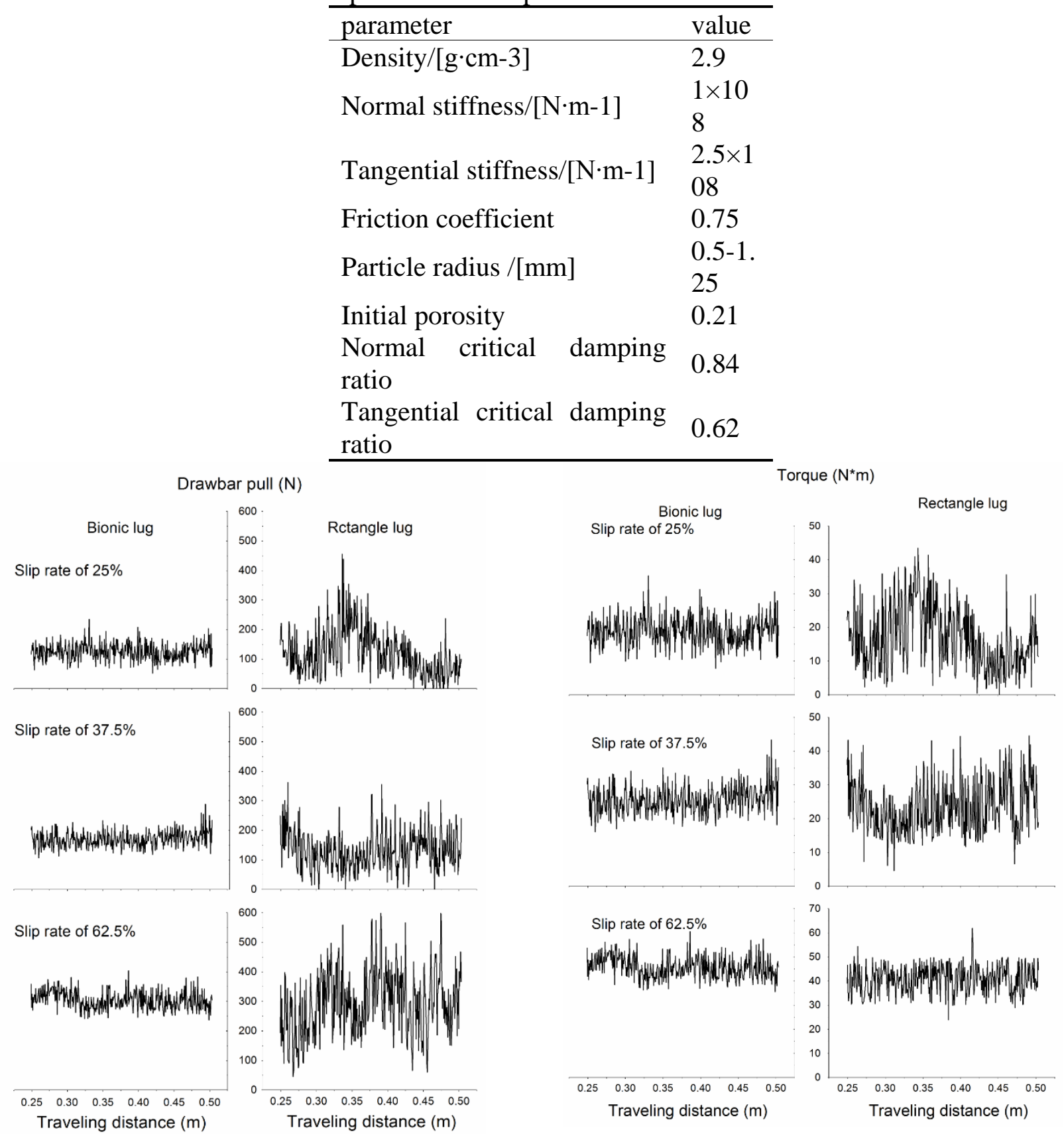

Fig. 5 Drawbar pull versus travelling distance

Fig. 6 Torque of the wheels versus travelling distance

As shown in Fig. 5, the drawbar pulls of both type of wheel were raising with the increasing of slip rate. The values of drawbar pulls fluctuates around the average value, which was a result of the unsmooth transition of wheel during rolling caused by the interval arrangement of the wheel lugs. The average drawbar pull of the wheel with bionic lugs were $125.7 \mathrm{~N}, 171.28 \mathrm{~N}$ and $244.19 \mathrm{~N}$ under the slippage rates $25 \%, 37.5 \%$ and $62.5 \%$. Although the average drawbar pull of the wheel with rectangle lugs was slightly smaller than that of the wheel with bionic lugs, the fluctuation of drawbar pull of the former one was considerably greater than that of the later one.

Under the slippage rate $25 \%, 37.5 \%$ and $62.5 \%$, the mean torque of the wheel with bionic lug were respectively $21.4 \mathrm{~N} \cdot \mathrm{m}, 29.9 \mathrm{~N} \cdot \mathrm{m}$ and $45.5 \mathrm{~N} \cdot \mathrm{m}$ (Fig. 6) and the mean torque of the wheel with rectangle lug were $20.9 \mathrm{~N} \cdot \mathrm{m}, 29.0 \mathrm{~N} \cdot \mathrm{m}$ and $38.6 \mathrm{~N} \cdot \mathrm{m}$. As shown in Fig. 6, the mean torque of the former wheel was far larger than the later at the slip rate of $62.5 \%$. The varying pattern of the curves 
of torques versus wheel displacement was basically similar with the curves of drawbar pull versus wheel displacement, because the torques was correlated directly with the drawbar pull.

\section{Summary}

Based on the theory of terramechanics, the computer aided design software CATIA and 3D DEM software PFC3D was combined by the interface codes and the embedded FISH language in PFC3D. This study established the dynamic simulation system of interactions between rigid wheel with irregular structure and loose lunar soil stimulants. The 3D simulation system was validated by the dynamic simulation analysis of interaction between rigid wheel and lunar soil simulants and comparing the simulated results with the laboratory test. At the same time, the wheel-soil interactions were simulated by the 3D wheel-soil simulation system. The results revealed that the bionic lug, compared with the rectangle lug, had large traction and a well stability.

\section{Acknowledgments}

The authors are grateful for the financial support by the National Natural Science Foundation of China (No. 51275199), the Science and Technology Research Project in the 12th Five-Year Period of Education Department of Jilin Province of China (Jijiaokehezi[2015] No. 204) and the Science and Technology Development Planning Project of Jilin Province of China (No. 20140101074JC).

\section{References}

[1] J.Y. Wong: Advances and Challenges in Terrain-Vehicle Systems - Presidential Address. Proceedings of the 15th International Conference of the Society for Terrain-Vehicle Systems. Hayama, Japan. Opening Session: 001(2005).

[2] Z. Zhang, J. Tong, D. Chen, J. Sun and Y. Ma: Modal analysis of bionic convex teeth rolling component composed of different materials. Transactions of the Chinese Society of Agricultural Engineering Vol. 28(13) (2012), p.8-15

[3] W. Ji, H. Jia and J. Tong. Experiment on working performance of bionic blade for soil-rototilling and stubble-breaking. Transactions of the Chinese Society of Agricultural Engineering Vol. 28(12) (2012), p.24-30

[4] J.Z. Li: Design and test of the bionic camel foot rubber tire for desert vehicles. Transactions of the Chinese Society of Agricultural Engineering Vol.15(2) (1999), p.32-36.

[5] P. A. Cundall and O.D.L. Strack: A discrete numerical method for granular assemblies. Geotechnique Vol. 29(1) (1979), p.47-65

[6] H. Nakashima, H. Fujii, A. Oida, M. Momozu , Y. Kawase, H. Kanamori, S. Aoki and T. Yokoyama, Parametric analysis of lugged wheel performance for a lunar microrover by means of DEM. Journal of Terramechanics Vol.44 (2007), p.153-162 